Introduction Patients with severe asthma are estimated to comprise $5-10 \%$ of the total asthma population but contribute disproportionately to the overall burden of disease. A growing body of evidence exists that implicates steroid exposure in morbidity and healthcare costs among this group.

Aim This study sought to quantify the additional healthcare costs associated with steroid exposure among patients with severe asthma.

Methods Data on patients severe asthma (GINA treatment step 5 with $\geq 4$ prescriptions/year oral corticosteroids, $\mathrm{n}=808$ ), was obtained from the Optimum Patient Care Research Database (OPCRD) database along with age and gender matched mild/ moderate asthma patients (GINA treatment step 2/3, n = 3975) and non-asthmatic controls (rhinitis only, $\mathrm{n}=1865$ ) Data included details of all scheduled and unscheduled healthcare consultations and details of prescribed medicines. Data on service use were extracted for the two most recent years for which observations were available. Healthcare contacts were monetised using unit costs extracted from the Personal Social Services Research Unit's reference costs and for drugs using Prescription Cost Analysis data. All costs were expressed in their 2013 equivalents. Sensitivity analyses related to identification of staff providing specific consultations or activity, and high/low estimates based on assumptions used were produced. Mean high/low healthcare costs over two years by group were estimated and compared as were costs estimated separately for healthcare contacts and prescribed medicines.

Results As shown in Table 1 mean per patient drug, healthcare activity and combined drug and activity costs were significantly higher for the severe asthma group relative to the mild/moderate group with asthma and the non-asthma controls in both high and low cost scenarios. The mean difference in combined cost between the severe and non-asthma controls groups was between $£ 5,031$ (low cost) and $£ 5,545$ (high cost) depending on the cost scenario and $£ 4,098$ (low cost) and $£ 4,510$ (high cost) compared to the mild asthma group.

\begin{tabular}{|c|c|c|c|}
\hline \multirow{2}{*}{$\begin{array}{l}\text { Abstract P158 Table } 1 \\
\text { over } 2 \text { years }\end{array}$} & \multicolumn{3}{|c|}{ Mean (SD) per patient healthcare costs } \\
\hline & $\begin{array}{l}\text { Severe } \\
\text { asthma } \\
(n=808)\end{array}$ & $\begin{array}{l}\text { Mild/moderate } \\
\text { asthma } \\
(\mathrm{n}=3975)\end{array}$ & $\begin{array}{l}\text { Non-asthma } \\
\text { controls (rhinitis } \\
\text { only) } \\
(\mathrm{n}=1994)\end{array}$ \\
\hline Prescribed medicines (Mean) & $£ 3447$ & f972 & f514 \\
\hline (SD) & f5606 & f1619 & f1178 \\
\hline Activity- Low cost (Mean) & £4229 & $£ 2606$ & $£ 2131$ \\
\hline (SD) & f4296 & $£ 3267$ & $£ 2807$ \\
\hline Total - Low cost (Mean) & f7676 & $£ 3578$ & $£ 2645$ \\
\hline (SD) & f7490 & f3899 & £3254 \\
\hline Activity - High cost (Mean) & f5085 & f3051 & f2474 \\
\hline (SD) & f5364 & £4225 & f3529 \\
\hline Total - High cost (Mean) & f8533 & f4023 & f2988 \\
\hline (SD) & f8206 & f4791 & f3924 \\
\hline
\end{tabular}

Conclusions Patients with severe asthma matched by age and gender have significantly greater direct healthcare costs compared to patients with mild/moderate asthma and non-asthmatic subjects.

\section{P159 THE USE OF OMALIZUMAB IN SEVERE ASTHMA IS ASSOCIATED WITH A DECLINE IN BLOOD EOSINOPHILS}

${ }^{1} \mathrm{LJ}$ Holmes, ${ }^{2} \mathrm{G}$ Tavernier, ${ }^{2} \mathrm{~S}$ Fowler, ${ }^{1} \mathrm{~K}$ Hince, ${ }^{2} \mathrm{RM}$ Niven. ${ }^{1}$ University Hospital of South Manchester, Manchester, UK; ${ }^{2}$ Institute of Inflammation and Repair, Manchester Academic Health Sciences Centre, University of Manchester and National Institute of Health Research Respiratory and Allergy Clinical Research Facility, University Hospital of South Manchester, Manchester, UK

\subsection{6/thoraxjnl-2015-207770.296}

Introduction/aim Omalizumab (Xolair) is a recombinant humanised monoclonal antibody licensed for the treatment of severe allergic asthma patients with IgE mediated disease. The mechanism of action of omalizumab is to bind to IgE preventing interaction with FCERI (high affinity receptors) upon the surface of mast cells and basophils, thereby reducing the activation of the inflammatory cascade and associated clinical symptoms. Although blood eosinophilia is a useful measure of airway inflammation, the correlation between blood eosinophils and omalizumab treatment has not been described outside clinical trial. Therefore, our aims were to identify whether the introduction of omalizumab is associated with a change in the blood eosinophil count within our cohort of patients with severe asthma.

Methods We collected measurements of blood eosinophils in patients maintained on omalizumab for periods 12 months pre and post, and (where available) 24 months pre and post initiation of therapy. We also investigated any correlation between blood eosinophils pre and post treatment versus clinical parameters including total $\mathrm{IgE}$ and omalizumab dose.

Results Complete data sets including 12-month blood eosinophil data were available for 49 of our current cohort of 92 patients on treatment. The mean blood eosinophil count dropped from $0.33 \times 10^{9}$ cells $/ 1($ IQR 0.37$) \times 10^{9}$ cells/l pre-treatment to 0.22 $\times 10^{9}$ cells/l (IQR 0.30$)$ post treatment $(p=<0.001)$.

Additionally, we widened the window to 24 months pre and post initiation of treatment, if blood eosinophil data was not available at 12 months. Data for 59 patients demonstrated similar significant reduction in mean blood eosinophil, $0.34 \times$ $10^{9} \mathrm{cells} / \mathrm{l}$ (IQR 0.34$)$ pre-treatment to $0.23 \times 10^{9}$ cells/l (IQR $0.29)(p=0.003)$.

No association was seen between the changes in mean blood eosinophil counts post treatment versus total monthly omalizumab dose and baseline total IgE.

Conclusion Our data demonstrates a significant reduction in blood eosinophils in patients who are receiving omalizumab treatment when comparing blood eosinophils at 12 and 24 months pre and post treatment. Future work should investigate whether this is a direct result of omalizumab therapy, due to confounding by other treatment, or a marker of improved asthma control.

\section{P160 BONE PROTECTION IN ORAL CORTICOSTEROID DEPENDANT ASTHMATICS}

HH Hashim, PL Molyneaux, S Regan, JH Hull, A Menzies-Gow. Royal Brompton Hospital, London, UK

\subsection{6/thoraxjnl-2015-207770.297}

Introduction Asthmatics dependant on oral corticosteroids are at risk of bone loss and significant increase in fracture risk at the hip and spine. Current guidelines recommend assessment of bone mineral density and the need for bone protection in any patient exposed to glucocorticoids for over three months. We 
sought to assess the usage of bone protection in steroid dependant asthmatics.

Method A retrospective analysis of patients admitted for a systematic assessment of asthma over a 12 month period at the Royal Brompton hospital was performed. Steroid dependence was defined as daily maintenance oral glucocorticoid for over three months. Other Inflammatory conditions requiring corticosteroids resulted in study exclusion.

Results 151 patients were admitted for a systematic assessment over a 12 month period. The mean age of subjects at assessment was 46 years of age $( \pm 27 \mathrm{SD})$ and the majority were female (77\%). Fifty four subjects (36\%) were steroid dependent at the time of admission. The average daily dose of prednisolone prescribed was $12.5 \mathrm{mg}( \pm 5.9 \mathrm{SD})$. Two thirds of steroid dependent patients had been on corticosteroids for over 12 months (36/54).

At the time of referral 11 patients were on Bisphosphonates and 34 on vitamin D replacement. The systematic assessment encompassed a DEXA scan in 49 of the steroid dependent subjects; half demonstrated normal bone density (26/54), one third had osteopenia (16/54) and seven subjects had osteoporosis. Forty Six subjects had vitamin D levels checked and the mean levels were $48 \mathrm{nmol} / \mathrm{L}( \pm 27 \mathrm{SD})$.

Bisphosphonates were stopped in seven patients with normal bone mineral density (13\%), continued in 3 subjects and started during assessment in 4 . Vitamin D supplementation was continued in 30 subjects, stopped in 2 and started in 5 subjects with osteopenia and low vitamin D levels.

Conclusions In a cohort of oral glucocorticoids steroid dependant asthmatics over half have normal bone mineral density. While calcium and vitamin D supplementation occurs in the majority of subjects bisphosphonate are often used unnecessarily in a predominantly pre-menopausal and female population. Clearer guidelines for the investigation and monitoring of bone protection in steroid dependent asthmatics are required.

\section{Lung cancer management}

\section{P161 HOW HAS THE SURGICAL TREATMENT OF LUNG CANCER IN THE UK EVOLVED OVER THE LAST TWO DECADES? - AN ILLUSTRATIVE SURGEON'S EXPERIENCE}

R Bilancia, A Paik, A Sharkey, D Waller. Thoracic Surgery, Leicester, UK

\subsection{6/thoraxjnl-2015-207770.298}

Background/introduction The practice of lung cancer surgery in UK has seen many changes over the last 20 years, with innovations in surgical technique and investigatory modalities together with significant organisational changes.

Aims/objectives To assess how these changes have impacted on an individual Consultant surgical practice spanning this era.

Method We have retrospectively reviewed a single-surgeon practice from consultant appointment to the present (1997-2015) comprising 1708 consecutive lung cancer operations: 962 (56\%) lobectomy, 296 (17\%) sublobar resection, 250 (15\%) extended resection, 157 (9\%) pneumonectomy, 43 (3\%) open/close. Concurrently, 710 surgical staging procedures were performed. We analysed trends with time in type of procedure; open/close rates and in-hospital mortality.
Results 1557 anatomic resections were performed (87 cases/year, 67-130) with no significant decrease in the annual workload. There were significant changes in the types of surgical procedures performed over the time period: a significant decrease in pneumonectomy rate $(\mathrm{p}<0.001)$, mirrored by an increasing use of sleeve-resections ( $\mathrm{p}=0.088$ ); an increase in the proportion of anatomical resections by video assisted thoracic surgery (VATS) ( $p<0.001$ ), an overall increasing number of anatomical segmentectomies $(\mathrm{p}<0.001)$, with a stable rate of wedge resections (mean $6.3 \%, p=0.908$ ). There has been a significant decrease in surgical mediastinal staging, particularly after 2010 $(\mathrm{p}<0.001)$ with a significant reduction in the open/close rate, particularly after 2004 (4.8 vs. $0.65 \%, \mathrm{p}<0.001)$. Overall the in-hospital mortality rate has significantly decreased (from $7.1 \%$ in 1998 to $2.9 \%$ in $2015, \mathrm{p}=0.004$ ).
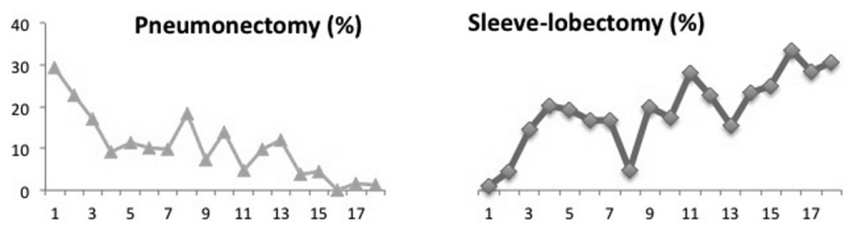

Anatomic Segmentectomy (\%)
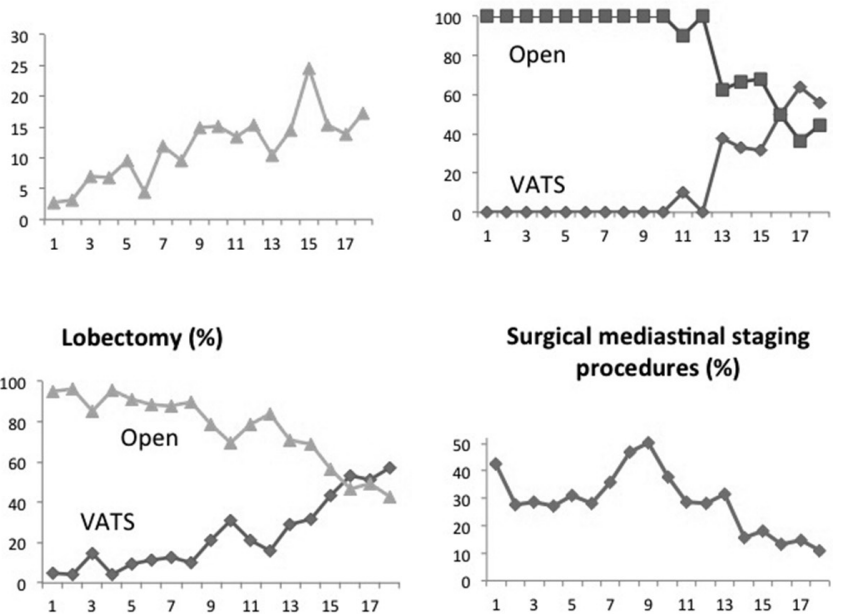

Surgical mediastinal staging procedures (\%)

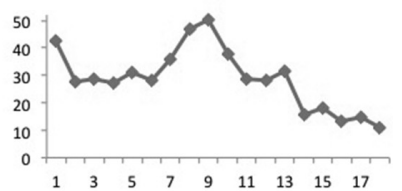

Open and close (\%)
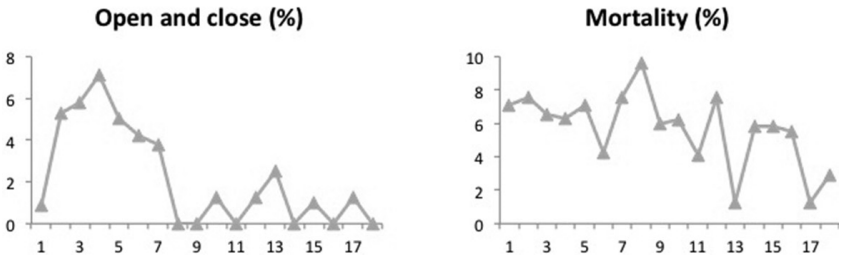

\section{Abstract P161 Figure 1}

Conclusion There has been significant evolution in lung cancer surgery over the last two decades, which are illustrated in this individual surgeon's practice. Whilst increased surgical experience may partly explain the changes, other important factors include: a change in the biology of lung cancer from central squamous to peripheral adenocarcinomas with earlier tumour detection, facilitating more VATS and lung-sparing surgery; improved perioperative care and the use of lesser resections, reducing mortality; and new techniques in staging (CT-PET, EBUS) reducing the need for surgical staging and the number of futile thoracotomies. 